\title{
A Simple Nonlinear Observer for State and Unknown Input Estimation: DC Motor Applications
}

\author{
Citlaly Martínez-García, Carlos Astorga-Zaragoza, Vicenç Puig, Juan Reyes-Reyes and Francisco López-Estrada
}

\begin{abstract}
The contribution of this work is to propose a method based on a simple nonlinear observer with an easy-to-compute observer gain, in order to achieve both state and unknown input estimation. Since the structure of the original model of the process does not require any transformation, the nonlinear dynamics are preserved. This allows an accurate estimation of the states and the unknown inputs or faults. Two particular cases involving DC motors are illustrated in simulation and an experiment using a permanent magnet DC motor is presented.
\end{abstract}

Index Terms-Nonlinear observer, unknown input, fault, DC motor.

\section{INTRODUCTION}

From the point of view of automatic control, many problems involving DC motors must be solved in the design stage before prototyping (i.e. modeling [1], control design [2], state/parameter estimation [3]) or at the operation stage (i.e. fault diagnosis [4], fault tolerant control [5], prognosis [6]). When a DC motor operates, it is subject to different operating conditions (predictable or not) caused by exogenous variables, disturbances, noise or faults, which can be induced by many causes such as vibrations, friction, overload, and voltage variations. Most of these conditions or phenomena unrelated to the ideal behavior are collectively known as unknown inputs (UI) [7]-[9].

Typically an unknown input is an unmeasured signal affecting a dynamical system. Many times, the knowledge of these signals is crucial to predict, compensate or to monitor the consequences of them over the system dynamics. Several authors have shown that the observer-based approaches are the most successful methods used to estimate unknown inputs, e.g., [10]-[12]. For instance, the authors in [10] uses a high-order sliding mode observers for leak estimation in pipelines where pressure and input-output flow rate measures are available. The case of UI observers for linear parameter variant (LPV) systems is recently treated in [11], where the computation of the observer gains via linear matrix inequalities (LMI's) is not a trivial task. The authors in [12] propose a disturbance observer-based controller for a direct-drive servo control system. In [5] and [13] UI observers are proposed for fault and state estimation for Takagi-Sugeno (T-S) fuzzy systems. In these cases, the observer design

Corresponding author: Carlos Astorga-Zaragoza. Department of Electronic Engineering, Tecnológico Nacional de México / CENIDET, Cuernavaca, Mor., 62490 Mexico, e-mail: astorga@cenidet.edu.mx

C. Martínez-García, J. Reyes-Reyes and F. López-Estrada are with Tecnológico Nacional de México / CENIDET / I.T. de Tuxtla Gutiérrez.

Vicenç Puig is with Advanced Control Systems (SAC) Research Group at Institut de Robòtica i Informàtica Industrial (IRI), Universitat Politècnica de Catalunya-BarcelonaTech (UPC), Barcelona, Spain. requires to transform the original nonlinear representation of the system into a T-S representation.

The contribution of this work is focused on proposing a method based on a simple nonlinear observer for state and unknown input estimation in DC motor applications (Section III). Two particular cases (Section II) for state and UI estimation involving DC motors are illustrated in simulation (Section IV). In order to demonstrate the practical applicability of the proposed approach, an experiment using a permanent magnet DC motor is presented in Section V.

\section{PRoblem StATEMEnT}

Two problems associated with unknown input estimation in DC motors are presented. For this end, two different mathematical models are selected from literature and used in this study: (1) a DC motor controlling an inverted pendulum [13] and (2) a permanent magnet DC motor [14].

1) Case 1. DC motor controlling an inverted pendulum: Consider the model of a DC motor controlling an inverted pendulum via gear train [13].

$$
\begin{aligned}
& \dot{x}_{1}=x_{2} \\
& \dot{x}_{2}=\frac{g}{l} \sin \left(x_{1}\right)+\frac{N K_{m}}{m l^{2}} x_{3} \\
& \dot{x}_{3}=-\frac{K_{b} N}{L_{a}} x_{2}-\frac{R_{a}}{L_{a}} x_{3}+\frac{1}{L_{a}} u
\end{aligned}
$$

where $x_{1}=\varphi_{p}$ is the motor shaft position, $x_{2}=w_{p}$ is the motor shaft velocity, $x_{3}=I_{a}$ is the armature current, $u$ is the voltage input. $K_{m}=0.1 \mathrm{Nm} / A$ is the motor torque constant, $K_{b}=0.1 \mathrm{Vs} / \mathrm{rad}$ is the back emf, $g=9.8 \mathrm{~m} / \mathrm{s}^{2}$ is the gravity, $N=10$ is the gear ratio, $l=1 m$ is the shaft length, $m=1 \mathrm{~kg}$ is the mass, $R_{a}=1 \Omega$ is the armature resistance and $L_{a}=$ $0.1 H$ is the armature inductance.

Assume that there is an unknown additive fault $\Delta u$ in the input of system (1). In such a case, the input $u$ is replaced by $u+\Delta u$, where $\Delta u$ can be an undesired variation of the voltage input which needs to be estimated in order to compensate it. Besides, assume that the motor shaft velocity $w_{p}$ and the armature current $I_{a}$ are not measured.

Assumption 1. The voltage input variation $\Delta u$ is unknown.

Assumption 2. The shaft position $x_{1}=\varphi_{p}$ is measurable.

Assumption 1 implies that although the process input $u$ is assumed to be known, actuators that provide this signal can be affected by faults $\Delta u$, i.e. non measurable signals. Assumption 
2 implies that $x_{1}$ is the system output, i.e. $y=x_{1}$. Then, system (1) can be represented as:

$$
\begin{aligned}
& \dot{x}=A x+g(y, u)+F \theta \\
& y=C x
\end{aligned}
$$

where $x=\left(\begin{array}{lll}x_{1} & x_{2} & x_{3}\end{array}\right)^{T}=\left(\begin{array}{lll}\varphi_{p} & w_{p} & I_{a}\end{array}\right)^{T}, y=x_{1}=\varphi_{p}$, $\theta=\Delta u$ and

$$
\begin{gathered}
A=\left(\begin{array}{ccc}
0 & 1 & 0 \\
0 & 0 & \frac{N K_{m}}{m l^{2}} \\
0 & -\frac{K_{b} N}{L_{a}} & -\frac{R_{a}}{L_{a}}
\end{array}\right), g(y, u)=\left(\begin{array}{c}
0 \\
\frac{g}{l} \sin x_{1} \\
\frac{1}{L_{a}} u
\end{array}\right), \\
C=\left(\begin{array}{lll}
1 & 0 & 0
\end{array}\right), \quad F=\left(\begin{array}{ll}
0 & 0 \frac{1}{L_{a}}
\end{array}\right)^{T} .
\end{gathered}
$$

The authors in [13] propose a Takagi-Sugeno observer in order to estimate the states and faults simultaneously. However, the computation of the gains is not a trivial task. In the following sections, an alternative method for state and unknown input estimation is proposed.

2) Case 2. Permanent magnet DC motor: Consider the following model of a permanent magnet DC motor [14].

$$
\begin{aligned}
\dot{i}_{a} & =-\frac{R_{a}}{L} i_{a}-\frac{K_{e}}{L} v_{m}+\frac{1}{L} u \\
\dot{v}_{m} & =\frac{K_{T}}{J_{1}} i_{a}-\left(\frac{f_{r}-f_{p} v_{m}}{J_{1}}\right) v_{m}-\frac{T_{0}-T_{2}}{J_{1}}
\end{aligned}
$$

where $i_{a}$ is the armature current, $v_{m}$ is the rotational speed (rpm), $u$ is the armature voltage (the input of the system), $T_{0}$ and $T_{2}$ are respectively the no-load and load torque. $R_{a}=1 \Omega$ is the armature resistance, $L=0.9 H$ is the inductance, $K_{e}=13.5 \times 10^{-3} \mathrm{~V} / \mathrm{rpm}$ is the back-emf coefficient, $K_{T}=0.1 \mathrm{Nm} / \mathrm{A}$ is the torque-current coefficient, $J_{1}=66.9 \times 10^{-4} \mathrm{Nms}$ is the normalized inertial moment, $f_{r}=1.4 \times 10^{-4} \mathrm{Nm} / \mathrm{rpm}$ is the friction coefficient (lubrication), $f_{p}=7.5 \times 10^{-8} \mathrm{Nm} / \mathrm{rpm}^{2}$ is the friction coefficient (aerodynamics) and $T_{2}=22.9 \times 10^{-2} \mathrm{Nm}$ is the load torque.

Assumption 3. The rotational speed $v_{m}$ and the armature voltage $u$ are measurable.

Assumption 3 implies that $v_{m}$ and $u$ are two known signals, i.e. they do not need to be estimated. Besides, $v_{m}$ is the measured output of the system, i.e. $y=v_{m}$. It should be noted that the armature current $i_{a}$ and the no-load torque $T_{0}$ are unknown and they need to be estimated. Then, system (5) has the form of system (2), where $x=\left(\begin{array}{ll}x_{1} & x_{2}\end{array}\right)^{T}=\left(\begin{array}{ll}i_{a} & v_{m}\end{array}\right)^{T}$, $y=x_{2}=v_{m}, \theta=T_{0}$ and

$$
\begin{gathered}
A=\left(\begin{array}{cc}
-\frac{R_{a}}{L} & 0 \\
\frac{K_{T}}{J_{1}} & 0
\end{array}\right), C=\left(\begin{array}{ll}
0 & 1
\end{array}\right), F=\left(\begin{array}{c}
0 \\
-\frac{1}{J_{1}}
\end{array}\right), \\
g(y, u)=\left(\begin{array}{c}
-\frac{K_{e}}{L} v_{m}+\frac{1}{L} u \\
-\left(\frac{f_{r}-f_{p} v_{m}}{J_{1}}\right) v_{m}+\frac{T_{2}}{J_{1}}
\end{array}\right)
\end{gathered}
$$

\section{THE PROPOSED OBSERVER}

Consider a nonlinear system having the following form:

$$
\begin{aligned}
\dot{x} & =A x+g(y, u)+F \theta \\
y & =C x
\end{aligned}
$$

where $x \in \mathbb{R}^{n}$ is the state of the system, $g(y, u) \in \mathbb{R}^{n}$ is a vector field depending on the system input $u$ and the output $y$. $A, F$ and $C$ are constant matrices of appropriate dimensions and $\theta \in \mathbb{R}^{d}$ is an unknown constant or slow-varying input, i.e. $\dot{\theta} \approx 0$.

\section{Assumption 4. The following rank conditions are satisfied}

$$
\text { - } \operatorname{rank}(F)=d \text {. }
$$

$$
\text { - } \operatorname{rank}(O)=n \text {, where } O=\left(\begin{array}{l}
C \\
C A \\
\ldots \\
C A^{n-1}
\end{array}\right)
$$

The first rank condition $\operatorname{rank}(F)=d$ in Assumption 4 implies that vector $\theta \in \mathbb{R}^{d}$ can be estimated. On the other hand, $\operatorname{rank}(O)=n$, implies that system (8) is observable.

Theorem 1. Assume that Assumption 4 holds. A nonlinear Luenberger-like observer for state and unknown input estimation for nonlinear systems having the form in Eq. (2) is:

$$
\begin{aligned}
\dot{\hat{x}} & =A \hat{x}+g(y, u)+F \hat{\theta}+K_{1}(y-\hat{y}) \\
\dot{\hat{\theta}} & =K_{2}(y-\hat{y}) \\
\hat{y} & =C \hat{x}
\end{aligned}
$$

if there exist two matrices $K_{1}$ and $K_{2}$ such that $A_{s}=$ $\left(\begin{array}{ll}A-K_{1} C & F \\ -K_{2} C & 0\end{array}\right)$ is a stability matrix.

Proof. Define the estimation errors $e_{x}=x-\hat{x}$ and $e_{\theta}=\theta-\hat{\theta}$. The time derivative of $e_{x}$ and $e_{\theta}$ are, respectively:

$$
\begin{gathered}
\dot{e}_{x}=\dot{x}-\dot{\hat{x}}=\left(A-K_{1} C\right) e_{x}+F e_{\theta} \\
\dot{e}_{\theta}=\dot{\theta}-\dot{\hat{\theta}}=-K_{2} C e_{x}
\end{gathered}
$$

Eqs. (10) and (11) can be written in a compact form as

$$
\dot{e}=A_{s} e
$$

where:

$$
A_{s}=\left(\begin{array}{ll}
A-K_{1} C & F \\
-K_{2} C & 0
\end{array}\right)
$$

and $e=\left(\begin{array}{ll}e_{x} & e_{\theta}\end{array}\right)$. If $K_{1}$ and $K_{2}$ are selected in such a way that $A_{s}$ is a stable matrix, then system (12) is stable, i.e. the observer converges.

Matrix (13) can be rewritten as:

$$
A_{s}=(\mathbb{A}-\mathbb{K} \mathbb{C})
$$

where

$$
\mathbb{A}=\left(\begin{array}{cc}
A & F \\
0 & 0
\end{array}\right) \mathbb{K}=\left(\begin{array}{c}
K_{1} \\
K_{2}
\end{array}\right) \mathbb{C}=\left(\begin{array}{ll}
C & 0
\end{array}\right)
$$

Eq. (14) can easily be solved to compute the observer gain vector $\mathbb{K}$ by any pole placement algorithm. Asymptotic convergence of the observer, i.e. $\lim _{t \rightarrow \infty} e=0$ is guaranteed if the eigenvalues of matrix $A_{s}$ have positive real part, where $e$ is the estimation error in Eq. (12). 


\section{OBSERVER DESIGN AND SIMULATION RESULTS}

In this section, the proposed observer in Section III is used for state and UI estimation of the processes described in Section II.

1) Case 1. DC motor controlling an inverted pendulum: Consider the system given in Eq. (2).

Remark 1. It should be noted that $\theta \in \mathbb{R}^{1}$ and $x \in \mathbb{R}^{3}$, then $d=1$ and $n=3$. In consequence Assumption 4 is verified, i.e. $\operatorname{rank}(F)=1, \operatorname{rank}(O)=3$, where

$$
O=\left(\begin{array}{l}
C \\
C A \\
C A^{2}
\end{array}\right)
$$

Then, an observer having the form (9) is designed to estimate the unmeasured states and the unknown input $\Delta u$ :

$$
\begin{aligned}
\dot{\hat{x}} & =\left(\begin{array}{ccc}
0 & 1 & 0 \\
0 & 0 & \frac{N K_{m}}{m l^{2}} \\
0 & -\frac{K_{b} N}{L_{a}} & -\frac{R_{a}}{L_{a}}
\end{array}\right) \hat{x}+\left(\begin{array}{c}
0 \\
\frac{g}{l} \sin x_{1} \\
\frac{1}{L_{a}} u
\end{array}\right) \\
& +\left(\begin{array}{c}
0 \\
0 \\
1 \\
L_{a}
\end{array}\right) \hat{\theta}+K_{1}(y-\hat{y}) \\
\dot{\hat{\theta}} & =K_{2}(y-\hat{y}) \\
\hat{y} & =C \hat{x}=\hat{\varphi}_{p}
\end{aligned}
$$

where matrices $K_{1}$ and $K_{2}$ are computed by pole placement of the stability matrix $A_{s}$ given in Eq. (13).

A numerical simulation is carried out in MATLAB ${ }^{(c)}$ to show the performance of the observer. If the eigenvalues of matrix $A_{s}$ are located at $\lambda_{1}=2.2, \lambda_{2}=2.8, \lambda_{3}=2.9$ and $\lambda_{4}=4$, then $K_{1}=\left(\begin{array}{lll}1.90 & 23.26 & -151.09\end{array}\right)$, $K_{2}=7.14$. The initial conditions of the model and the observer are: $x^{0}=\left(\begin{array}{llll}\varphi_{p}^{0} & w_{p}^{0} & I_{a}^{0}\end{array}\right)^{T}=\left(\begin{array}{lll}0.01 & 0 & 0\end{array}\right)^{T}$, $\hat{x}^{0}=\left(\begin{array}{lll}\hat{\varphi}_{p}^{0} & \hat{w}_{p}^{0} & \hat{I}_{a}^{0}\end{array}\right)^{T}=\left(\begin{array}{lll}0.065 & 0.215 & 0.12\end{array}\right)^{T}$ and $\hat{\theta}^{0}=0$. The input voltage $u=1.5 \mathrm{~V}$. Eqs. (5) and (16) are integrated using a Runge-Kutta first order method (Euler) with an integration step $T_{s}=0.05 \mathrm{~s} . \Delta u$ is simulated as follows:

$$
\theta=\Delta u=\left\{\begin{array}{cl}
0 \mathrm{~V}, & 0 s \leq t<35 \mathrm{~s} \\
0.5 \mathrm{~V}, & 35 \mathrm{~s} \leq t<75 \mathrm{~s} \\
0.8 \mathrm{~V}, & 75 \mathrm{~s} \leq t
\end{array}\right.
$$

As displayed in Fig. 1 the estimated states $\hat{x}_{2}=\hat{w}_{p}$ and $\hat{x}_{3}=\hat{I}_{a}$ (dotted lines) asymptotically converge to the corresponding simulated values $x_{2}=w_{p}$ and $x_{3}=I_{a}$ (solid lines), despite the increments in the input $\Delta u$ (see Fig. 2) at $t=35 \mathrm{~s}$ and $t=75 \mathrm{~s}$. The motor shaft position $x_{1}=\varphi_{p}$ is not displayed because it is the measured variable and it does not need to be estimated. Fast convergence for state $x_{2}$ can be appreciated, whereas a convergence time of about $5 \mathrm{~s}$ is required to estimate state $x_{3}$ after each voltage increment in $\Delta u$. Convergence time can be modified/improved by selecting different stable eigenvalues of matrix $A_{s}$. On the other hand, the estimation of $\Delta \hat{u}$ is depicted in Fig. 2 (dotted line). As expected, the observer asymptotically converges to the simulated unknown input $\Delta u$ (solid line).
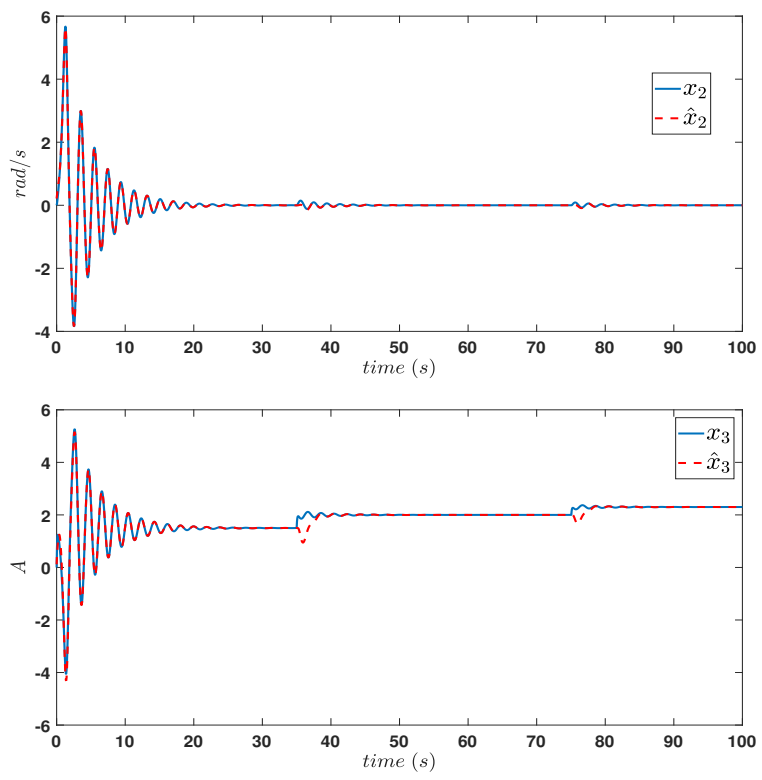

Fig. 1. Estimation of the states $\varphi_{p}, w_{p}$ and $I_{a}$ in Case 1.

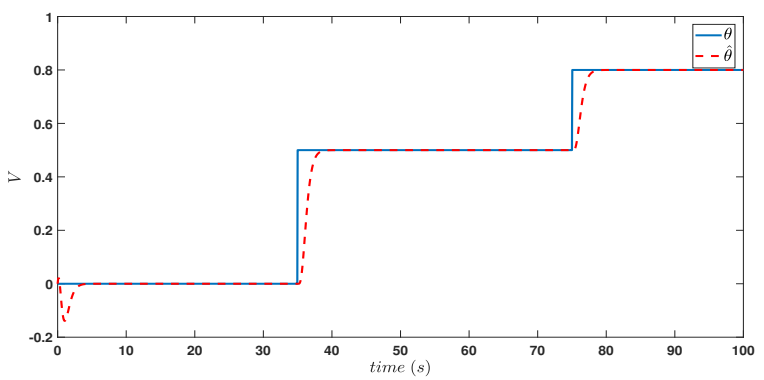

Fig. 2. Estimation of the UI $\theta=\Delta u$ in Case 1 .

2) Case 2. Permanent magnet DC motor: Consider the system given in Eq. (2) and described by matrices given in (6)-(7).

Remark 2. It should be noted that $\theta \in \mathbb{R}^{1}$ and $x \in \mathbb{R}^{2}$, then $d=1$ and $n=2$. In consequence Assumption 4 is verified, i.e. $\operatorname{rank}(F)=1, \operatorname{rank}(O)=2$, where $O=\left(\begin{array}{l}C \\ C A\end{array}\right)$.

By considering Remark 2, an observer having the form of system (9) can be designed to estimate the unmeasured state $i_{a}$ and the unknown input $T_{0}$ as follows:

$$
\begin{aligned}
\dot{\hat{x}} & =\left(\begin{array}{cc}
-\frac{R_{a}}{L} & 0 \\
\frac{K_{T}}{J_{1}} & 0
\end{array}\right) \hat{x}+\left(\begin{array}{c}
-\frac{K_{e}}{L} y+\frac{1}{L} u \\
-\left(\frac{f_{r}-f_{p} y}{J_{1}}\right) y+\frac{T_{2}}{J_{1}}
\end{array}\right) \\
& +\left(\begin{array}{c}
0 \\
-\frac{1}{J_{1}}
\end{array}\right) \hat{\theta}+K_{1}(y-\hat{y}) \\
\dot{\hat{\theta}} & =K_{2}(y-\hat{y}) \\
\hat{y} & =C \hat{x}
\end{aligned}
$$

where the observer gains $K_{1}$ and $K_{2}$ are constant matrices of 
appropriate dimensions. These matrices are easily computed by pole placement of the stability matrix $A_{s}$ given in Eq. (13).

A numerical simulation is carried out in MATLAB ${ }^{\complement}$ in order to show the observer performance for state and unknown input estimation, i.e. the armature current $i_{a}$ and the no-load torque $T_{0}$. The process model (5) and the observer (16) are simulated using the parameter values given in section II. If the eigenvalues of matrix $A_{s}$ in Eq. (13) are required to be located at $\lambda_{1}=0.41, \lambda_{2}=0.42$ and $\lambda_{3}=0.40$, then the observer gains are: $K_{1}=\left(\begin{array}{ll}0.027 & 0.030\end{array}\right), K_{2}=-3.84 \times 10^{-4}$. The initial conditions are $x^{0}=\left(\begin{array}{ll}i_{a}^{0} & v_{m}^{0}\end{array}\right)^{T}=\left(\begin{array}{ll}4 & 0.02\end{array}\right)^{T}$, $\hat{x}^{0}=\left(\begin{array}{ll}\hat{i}_{a}^{0} & \hat{v}_{m}^{0}\end{array}\right)^{T}=\left(\begin{array}{ll}2 & 0.10\end{array}\right)^{T}$ and $\hat{\theta}^{0}=0.04$. The input voltage $u=10 \mathrm{~V}$. Integration of Eqs. (5) and (16) are performed using a Runge-Kutta first order method (Euler) with an integration step of $0.01 \mathrm{~s}$. Two step changes of the no-load torque $T_{0}$ are considered as follows:

$$
T_{0}=\left\{\begin{array}{l}
0.023 \mathrm{Nm}, \quad 0 s \leq t<50 \mathrm{~s} \\
0.040 \mathrm{Nm}, \quad 50 \mathrm{~s} \leq t<100 \mathrm{~s} \\
0.050 \mathrm{Nm}, \quad 100 \mathrm{~s} \leq t
\end{array}\right.
$$

Simulation results are depicted in Figs. 3-4. In Fig. 3, it can be appreciated that the estimated current $\hat{i}_{a}$ (dotted lines) asymptotically converges to the simulated process variable $i_{a}$ (solid line). The rotational speed $x_{2}=v_{m}$ is not displayed because it is the measured variable and it does not need to be estimated. Besides, the estimated no-load torque $\hat{\theta}=\hat{T}_{0}$, (dotted line in Fig. 4) converges even if the simulated value $\theta=T_{0}$ (solid line in Fig. 4) undergoes significant step changes.

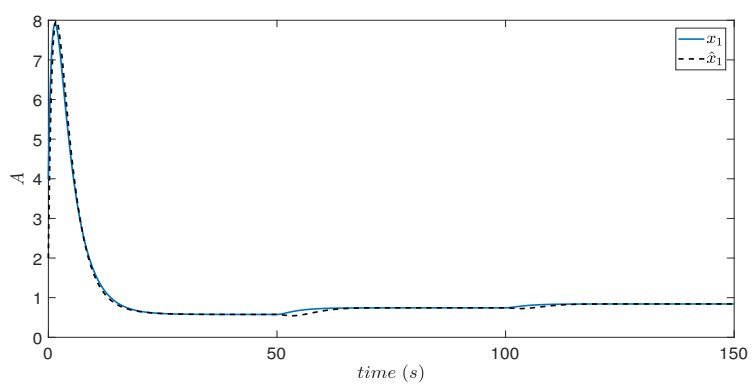

Fig. 3. Estimation of $x_{1}=i_{a}$ in Case 2 .

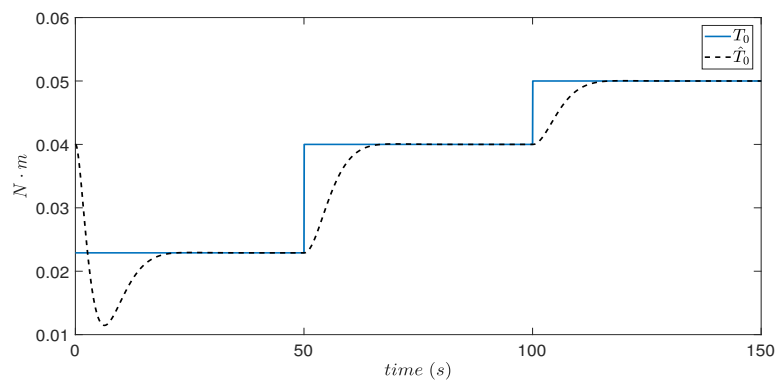

Fig. 4. Estimation of the unknown input $\theta=T_{0}$ in Case 2.

\section{EXPERIMENTAL RESULTS}

Several experiments were carried out in a laboratory prototype for estimation of the armature current $i_{a}$ and the no-load torque $T_{0}$. A typical operation is presented in this section.

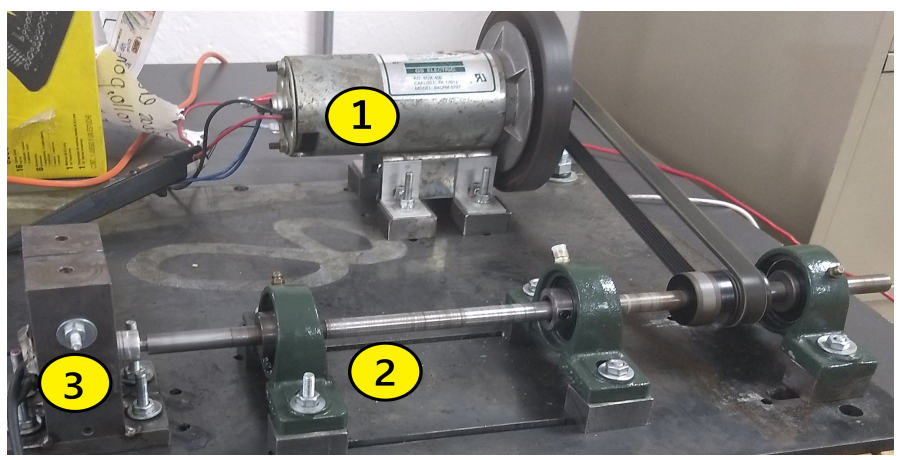

Fig. 5. Laboratory prototype. DC motor connected to a bearing train.

The prototype consists of a DC motor (labeled as 1 in Fig. 5) coupled to a bearing train (labeled as 2 in Fig. 5). The physical parameters according to the employed DC motor are given in Section II, Case 2. These parameters were obtained by direct physical measurements, or they were approximately deduced and verified through laboratory experiments.

The rotational speed of the shaft $v_{m}$ was measured by coupling a 1000 pulses per revolution incremental encoder (Koyo TRD-S1000-VD, labeled as 3 in Fig. 5). The frequency signal provided by this encoder was acquired and treated by a NI myRIO-1900 card with a sampling time $T_{s}=1 \mu \mathrm{s}$ to deliver a proportional signal to the speed of the motor in r.p.m. The voltage input, provided by a Magna-Power programmable DC power supply was constant at $u=15 \mathrm{~V}$.

Assume (as in the previous simulation) that the armature current $i_{a}$ is not measured and the no-load torque $\theta=T_{0}$ is unknown. Then, the implemented observer has the form of system (16). The observer gains $K_{1}$ and $K_{2}$ are

$$
K_{1}=\left[\begin{array}{c}
0.02747 \\
0.33446 \times 10^{-4}
\end{array}\right] \quad K_{2}=-3.83967 \times 10^{-4}
$$

By using these gains, the observer stability matrix $A_{s}$, defined in Eq. (13), has suitable eigenvalues $\lambda_{1}=-0.63$ and $\lambda_{2,3}=-0.28 \pm 0.17 i$, ensuring an adequate observer convergence. The initial conditions of the observer are $\hat{x}^{0}=\left[\begin{array}{ll}1.4 & 700\end{array}\right]^{T}, \theta^{0}=\hat{T}_{0}^{0}=0.23$.

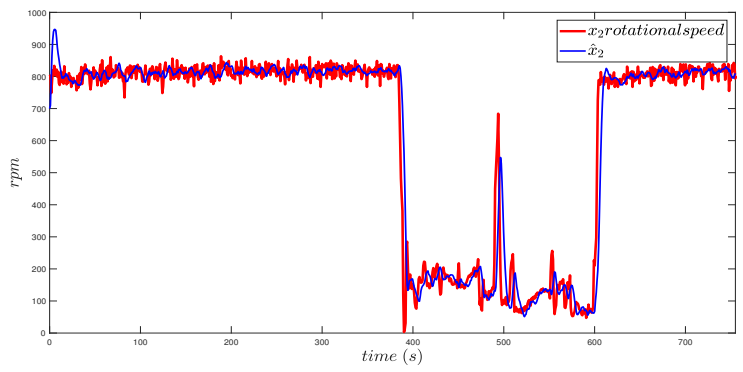

Fig. 6. Measurement and estimation of the rotational speed. 


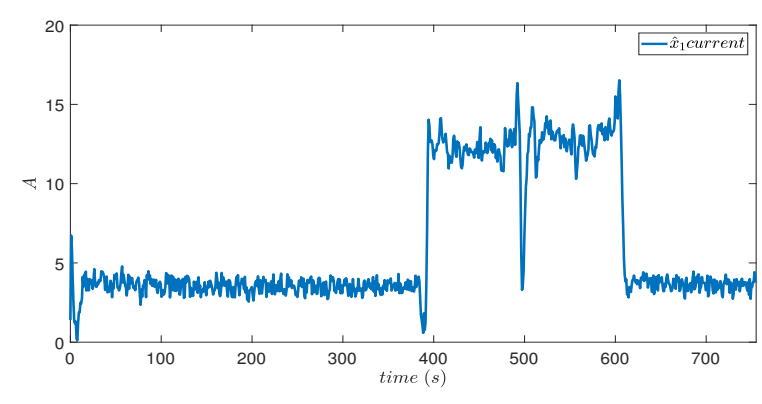

Fig. 7. Estimation of armature current.

The experiment was performed as follows. Once the motor speed reaches the steady-state value (around 800 r.p.m. as illustrated in Fig. 6), a mechanically-induced friction force is generated from around $t=390 \mathrm{~s}$ to $t=600 \mathrm{~s}$. This action emulates a faulty scenario, probably due to unhealthy bearings. In this way, a variation on the value of $T_{0}$ is expected.

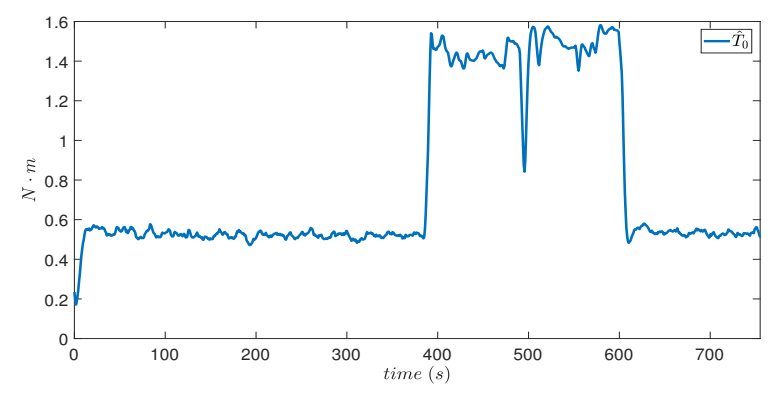

Fig. 8. Estimation of $T_{0}$.

Experimental results are depicted in Figs. 6-8. In Fig. 6 , it can be appreciated that the estimated rotational speed $\hat{x}_{2}=\hat{v}_{m}$ (blue line) asymptotically converges to the measured value (red line) despite the fault occurrence and measurement noise. As expected, the armature current $i_{a}$ increases when the friction force is induced. The corresponding estimated armature current $\hat{x}_{1}=\hat{i}_{a}$ is depicted in Fig. 7. Finally, the unknown input occurrence is reflected as an increasing variation in $T_{0}$, whose estimated value of $\hat{T}_{0}$ is depicted in Fig. 8.

\section{CONCLUSION}

A simple nonlinear observer for state and UI estimation is presented in this paper. Two cases are presented in order to illustrate its applicability to DC motors. Preliminary results in simulation demonstrate asymptotic convergence of the observer. Besides, a laboratory experiment is performed with a laboratory prototype, consisting in a DC motor coupled to a bearing supported shaft, where faults (like these appearing when bearings become faulty) are induced, to test the fault detection and estimation capabilities of the proposed observer. The two main advantages of the proposed observer, compared with [13], and similar works dealing with Takagi-Sugeno systems are: $(i)$ the easiness to compute the observer gain, because a simple pole placement procedure is required to calculate the observer gain and (ii) the mathematical model of the processes does not require any transformation, preserving the original nonlinear dynamics of the model. Thus, an accurate estimation of the non-measurable states and the unknown inputs and faults can be guaranteed. On the other hand, the main limitation of this observer is that it can only be applied for nonlinear systems having the form of system (8) where all variables in the nonlinear vector $g(y, u)$ must be measurable. Fortunately, in practice, many systems can be modeled in this form.

\section{ACKNOWLEDGMENT}

This work was supported by CONACyT \& Tecnológico Nacional de México. The authors gratefully acknowledge Omar J. Martínez-Martínez and Héctor García-Ramírez for their support in the development of the laboratory prototype.

\section{REFERENCES}

[1] M. Korkosz, P. Bogusz, J. Prokop, "Modelling and experimental research of fault-tolerant dual-channel brushless DC motor", IET Electric Power Applications, vol. 12, no. 6, pp. 787-796, 2018.

[2] Y. Song, Z. Wang, D. Ding, and G. Wei, "Robust model predictive control under redundant channel transmission with applications in networked DC motor systems", Int. J. of Robust and Nonlinear Control, vol. 26, no. 18, pp. 3937-3957, 2016.

[3] J. Jiménez, F. Beltrán, F. González, and I. López, "On model parameter estimation methods of DC electric motors", Pistas Educativas, vol. 36, no. $112,2018$.

[4] S. Munikoti, L. Das, B. Natarajan, and B. Srinivasan, "Data driven approaches for diagnosis of incipient faults in DC motors", IEEE Trans. on Ind. Informatics, 2019.

[5] J. Lan, and R.-J. Patton, "Integrated design of fault-tolerant control for nonlinear systems based on fault estimation and T-S fuzzy modeling", IEEE Trans. on Fuzzy Systems, vol. 25, no. 5, pp. 1141-1154,2017.

[6] H. Sanchez, T. Escobet, V. Puig, Vicenç and P. Odgaard, "Health-aware model predictive control of wind turbines using fatigue prognosis", Int. J. of Adaptive Control and Signal Proc., vol. 32, no. 4, pp. 614-627, 2018.

[7] N. Gao, M. Darouach, H. Voos, and M. Alma, "New unified $H_{\infty}$ dynamic observer design for linear systems with unknown inputs", Automatica, vol. 65, pp. 43-52, 2016.

[8] A.-F. Taha, J. Qi, J. Wang, and J.-H. Panchal, "Risk mitigation for dynamic state estimation against cyber attacks and unknown inputs", IEEE Trans. on Smart Grid, vol. 9, no. 2, pp. 886-899, 2018.

[9] S.-K. Pandey, S.-L. Patil, U.-M. Chaskar, and S.-B. Phadke, "State and disturbance observer based integral sliding mode controlled boost DC-DC converters", IEEE Trans. on Circuits Syst. II, DOI:10.1109/TCSII.2018.2888570, 2018.

[10] H.-A. Fernández, C. Verde, and J. Moreno, "High-order sliding mode observer for outflow reconstruction in a branched pipeline", 2018 IEEE Conf. on Control Tech. and Applications (CCTA), pp. 595-600, 2018.

[11] B. Marx, D. Ichalal, J. Ragot, D. Maquin, and S. Mammar, "Unknown input observer for LPV systems", Automatica, vol. 100, pp. 67-74, 2019.

[12] L. Yu, J. Huang, and S. Fei, "Robust switching control of the direct-drive servo control systems based on disturbance observer for switching gain reduction", IEEE Trans. on Circuits Syst. II, DOI:10.1109/TCSII.2018.2881244, 2018.

[13] V.-P. Vu, and T.-D. Do, "Fault/state estimation observer synthesis for uncertain T-S fuzzy systems", IEEE Access, vol. 7, pp. 358-369, 2019.

[14] L. Menini, C. Possieri, A. Tornambè, "Application of algebraic geometry techniques in permanent-magnet DC motor fault detection and identification”, European Journal of Control, vol. 25, pp. 39-50, 2015. 